\title{
Lithium as a Protective Factor against Dementia in Bipolar Patients-A Retrospective Cohort Study
}

\author{
Diana Paleacu Kertesz ${ }^{1,2 *}$, Meir Dvir², Shelly Tadjer3 ${ }^{3}$ Asaf Shellef ${ }^{2,3}$ \\ ${ }^{1}$ Neurology Service and Memory Clinic, Abarbanel Mental Health Center, Bat Yam, Israel \\ ${ }^{2}$ Sackler School of Medicine, Tel Aviv University, Tel Aviv, Israel \\ ${ }^{3}$ Department of Psychiatry, Abarbanel Mental Health Center, Bat Yam, Israel \\ Email: ^diana.paleacu@abr.health.gov.il
}

How to cite this paper: Kertesz, D.P., Dvir, M., Tadjer, S. and Shellef, A. (2016) Lithium as a Protective Factor against Dementia in Bipolar Patients-A Retrospective Cohort Study. Advances in Alzheimer's Disease, 5, 155163.

http://dx.doi.org/10.4236/aad.2016.54011

Received: September 25, 2016

Accepted: December 26, 2016

Published: December 29, 2016

Copyright (๑) 2016 by authors and Scientific Research Publishing Inc. This work is licensed under the Creative Commons Attribution International License (CC BY 4.0).

http://creativecommons.org/licenses/by/4.0/

(c) (i) Open Access

\begin{abstract}
Background: Controversial research has indicated potential neuroprotective qualities of lithium in the prevention of dementia, but it did not lead to a definite conclusion. Aim: We seek to examine whether lithium has a protective effect in an elderly population suffering from bipolar disorder. Patients and Methods: Of a retrospective cohort including 433 patient files that met inclusion criteria (age over 65 years and bipolar disease coded by ICD), 147 files contained enough relevant data. The patients' files were divided into lithium users (LU) for those who used lithium for at least a minimum of 3 months and non-users (LNU) for those who did not use lithium at all or used it for a period of less than 3 months. We searched for dementia incidence in these two groups. Results: 433 files were collected initially through a computerized search using ICD10 code for bipolar affective disorder. One hundred twenty three files were excluded due to age (younger than 65 years), 7 were excluded due to neurological background, and 49 were excluded due to either a secondary or a not strictly bipolar psychiatric diagnosis. One hundred and seven were excluded due to insufficient/uncertain data. The remainders of 147 files were included in the final analysis. Twenty five of the 147 bipolar patients were diagnosed with dementia (17\%). Amongst the $92 \mathrm{LNU}$ patients, 16 developed dementia (17.4\%) and of the $55 \mathrm{LU}$ patients, 9 developed dementia (16.4\%); Pearson's $\chi^{2}(1)=0.026, \mathrm{p}<0.05$. Our analysis showed no significant difference between the two groups. Conclusions: No cognitive protective qualities of lithium treatment in elderly bipolar patients were demonstrated by our study.
\end{abstract}

\section{Keywords}

Lithium, Dementia, Protection, Bipolar Disease 


\section{Introduction}

Lithium compounds, mainly lithium salts are considered a "veteran" drug is psychiatry since 1948, when they were first shown by J. Cade to be useful in the treatment of manic psychosis in bipolar patients. Since then lithium has been used mostly as a mood stabilizer for affective disorders, mainly bipolar disorder. It has been proved effective in preventing recurrent manic episodes in two meta analyses from 2004 and 2007, though inconclusive about its efficacy in preventing recurring depressive episodes [1] [2]. In the past 15 years, the possibility that lithium might have neuroprotective effects, mostly in research concerning Alzheimer's disease (AD) has been proposed.

$\mathrm{AD}$ is known for two pathological hallmarks: The formation of $\mathrm{A} \beta$ amyloid plaques and neurofibrillary tangles, which are made from hyper phosphorylated tau proteins. Laboratory research demonstrated the neuroprotective capabilities of lithium, by showing its effect on the accumulation of the two pathological entities. The main mechanism suggested that lithium inhibits the glycogen synthase kinase-3 (GSK-3), a kinase with a crucial role in the process of forming the amyloid plaques [3]. In AD mouse models and cell culture research, GSK-3 has been shown to phosphorylate the tau proteins, and having a role in creating the $\mathrm{A} \beta$ amyloid. Two inhibitory mechanisms have been identified: lithium as a direct competitive inhibitor of GSK-3 and an indirect inhibition by activation of the protein kinase $\mathrm{B}$ (PKB) also known as the Akt enzyme, which in turn inhibits GSK-3 [4]. A Drosophila model demonstrated a decrease toxicity of $\mathrm{A} \beta$ amyloid in flies when using lithium; it reduced mortality and improved behavior [5]. When compared with other potential neuroprotective agents used in common psychiatric practice such as antipsychotics, other mood stabilizers, antidepressants, anxiolytics, antihistamines, anticholinergics, melatonin and modafinil, lithium has been considered one of the 3 most promising drugs for neuroprotection against $\mathrm{AD}$ [6].

Various other mechanisms for lithium involvement in neuroprotection have been suggested. It has been shown that lithium raises the levels of brain derived neurotrophic factor (BDNF), it is involved in regulating the glutamate receptors and reduces the brain arachidonic acid cascade [4] [5] [6] [7]. However, the processes are not fully understood. Furthermore, the contribution of the GSK-3 and other mechanism to neuroprotection is uncertain.

The laboratory research was followed by several clinical researches, trying to ascertain the potential neuroprotective effect of lithium in humans [8]-[14]. Some were positive [9] [10] [11] [12] [14] and some negative [8] [13]. The positive (neuroprotective) studies will be reviewed here.

A small retrospective cohort study by Takeshi in 2006 showed no difference in dementia prevalence between lithium users and no lithium users [9]. However the statistical power of the research was small, and as such, doubtful.

In 2007, a research by Nunes et al. compared a group of elderly bipolar lithium users vs. bipolar lithium non users. Contrary to the previous researches, it showed a marked increase in dementia in lithium non users vs. lithium users (33\% vs. 5\%) [10]. The researchers concluded that lithium has a neuroprotective effect within the bipolar popu- 
lation. Again, the number of subjects (118 patients) was not large enough.

In 2008, a research by Kessing et al. using Danish health services databases, compared 16,000 lithium users to 1.5 million controls, along 10 years. The research also tried to count for the varying use of lithium, by comparing the number of lithium prescriptions. The research came to a few important conclusions: 1) Subjects who had one prescription had considerably higher risk of dementia than the general population. 2) With increasing subscriptions the rate of dementia was reduced up to the general population's level. 3) With long term use of lithium, (Indicated with 10 or more prescriptions) the risk rises again, but not to the original levels [11]. The researchers assumed that the first conclusion signified the increased risk of dementia for the population that will need lithium compared to the general population, but that within this group, it showed neuroprotective qualities. The research had significant statistical power, but it did compare between two widely different populations. It did not compare between lithium users versus lithium non users within the mood affective population itself.

In 2010, a follow up research by the same group tried to compare the use of various drug groups (Lithium, anticonvulsants, antidepressants and antipsychotics) within a bipolar population and their effect on the dementia rates. The number of prescriptions was taken into account again. Within the group of lithium users a marked decrease in dementia rates was shown for subjects who had two or more prescriptions compared to just one, whereas the other medications did not show this affect. The researchers concluded that lithium is neuroprotective. However, a direct comparison between bipolar lithium users versus lithium non users was not undertaken [12].

In 2011, another randomized controlled study by the Forlenza group compared lithium users with lithium non users, but this time in 41 patients with mild cognitive impairment rather than established AD. Another important difference from the previous research is the long duration -12 months versus 10 weeks. The tested values were the same, with an added criteria-whether the patients developed AD or not. (The Hampel study used patients already suffering from AD). Fewer patients in the lithium user group developed $\mathrm{AD}$, and have shown lessened cognitive impairment. They have also demonstrated a decreased level of CSF phosphorylated tau, where the lithium non users showed a slight increase. AD incidence was lower than in the lithium non users group. The researchers concluded that lithium does act as a neuroprotective agent in the early stages of cognitive decline, and that its protective effect requires long term use [14].

The various researches show disagreement whether lithium acts as a neuroprotective factor or not. The Kessing researches were the most exhaustive, but they either compare the affected population to the general population (in the first research), or did not compare between lithium users to lithium nonusers within the bipolar group (in the second research they compared between varying doses of lithium use within the lithium users group). The Nunen research did compare between lithium users and lithium non users within the bipolar population, but lacked statistical power.

The goal of this research is to discern whether lithium diminishes the prevalence of 
dementia in a narrow population-elderly patients with a strict bipolar diagnosis, in order to avoid possible selection confounders. It is a retrospective cohort study, using databases from the Abarbanel Psychiatric Hospital and affiliated outpatient clinics.

\section{Methods}

This is a retrospective cohort where all patient files were gathered from the archives (computerized and paper files) of the Abarbanel Mental Health Center, The Yaffo Mental Health Clinic and the Petah Tiqva Mental Health Clinic (both adjoined to the hospital). The files included a period of 10 years (between January 2003-December 2013). They were scanned and personal identifying information was erased by a research assistant who coded the files. The material itself was viewed in a blinded fashion, recorded, and analyzed by the one of the researchers (MD), in order to minimize exposure of personal patient information. The study was revised and approved by the local IRB (Institutional Helsinky Committee).

\subsection{Inclusion and Exclusion Criteria}

We included patients who were 65 years old and above in the last entry of their files (this age was selected based upon the observation that incidence of dementia of the Alzheimer's type rises in populations older than 65 years old, and should be easily detected in this age group), and had an ICD-10 diagnosis of Bipolar disorder. In order to minimize confounders, we did not include any other affective disorder, like schizoaffective disorders and similar. We excluded patients who had a diagnosis of other psychiatric or neurological illnesses. We also excluded files for which there was uncertainty as to their diagnosis, age below 65 years old, uncertain status concerning lithium use or less than 3 months of continuous use.

\subsection{Tested Criteria}

We divided the files into 2 groups-Lithium users and lithium non users. "Lithium use" was defined as a record of at least 3 months of lithium use in the patient's file. The file was then examined for a diagnosis of dementia. A patient was diagnosed with dementia as defined by the DSM IV criteria. MMSE tests were not included in most of the files, and thus not included in the research either. We have also recorded age, gender, and when available-level of education (academic $\geq 12$ years, high school $\geq 10-12$ years and elementary school $=$ up to 10 years of education).

\subsection{Statistical Analysis}

We analyzed the data using the Pearson's $\chi^{2}$ for the categorical parameters. We then sought to do a Logistic regression for coupled parameters.

\section{Results}

\subsection{Research Population}

433 files were collected initially, through a search of a bipolar diagnosis. 123 were ex- 
cluded due to age (Under 65), 7 were excluded to neurological background, 49 were excluded due to either a secondary or a not strictly bipolar psychiatric diagnosis and 107 were excluded due to insufficient/uncertain data. The remainders of 147 files were included in the final analysis. The data regarding education levels were missing to a great degree (Only 89/147 patientshad information in that regard) and the results were not significant. Thus, they are not included (Figure 1).

The 147 patients had a mean age of 72.12 years (SD 6.23) range: 65 - 91 years. Eighty seven of them were women (59.2\%) and 40 were men (40.8\%).

\subsection{Lithium Use and Incidence of Dementia}

Fifty five of them used lithium (37.41\%) and 92 did not (62.58\%). Of the 147 patients, 25 were diagnosed with dementia (17\%).

Amongst the 92 patient who were not lithium users, 16 patients developed dementia (17.4\%) and of the 55 patients who were lithium users 9 patients developed dementia $(16.4 \%)$. Pearson's $\chi^{2}(1)=0.026, p>0.05$. There is no significant difference between the groups. The results are summarized in Table 1.

\subsection{Gender Effect}

We analyzed the data in groups by gender, trying to discern a different response dependent on it. 33 men were not lithium users, and 7 (21.2\%) were diagnosed with dementia. The 27 lithium users men however had only 2 cases of dementia. But $\chi^{2}(1)=$ $2.220, \mathrm{p}>0.05$ The difference is insignificant.

Amongst the women an opposite trend was seen-amongst the 59 lithium non users, 9 had dementia (15.3\%), while amongst the 28 lithium users women 7 had dementia (25\%). But again the difference is not significant. $\chi^{2}(1)=1.202, \mathrm{p}>0.05$. The results are shown in Table 2 and Table 3.

Table 1. Lithium and dementia, total population.

\begin{tabular}{cccc}
\hline & Lithium non user & Lithium user & Total \\
\hline No Dementia & $76(51.7 \%)$ & $46(31.3 \%)$ & $122(83 \%)$ \\
Dementia & $16(10.88 \%)^{*}$ & $9(6.1 \%)^{*}$ & $25(17 \%)$ \\
Total & $92(62.58 \%)$ & $55(37.41 \%)$ & $147(100 \%)$ \\
\hline
\end{tabular}

${ }^{\star}$ Pearson's $\chi^{2}(1)=0.026$.

Table 2. Lithium and dementia, Men only.

\begin{tabular}{cccc}
\hline & Lithium non user & Lithium user & Total \\
\hline No Dementia & $26(43.33 \%)^{*}$ & $25(41.66 \%)^{*}$ & $51(85 \%)$ \\
Dementia & $7(11.66 \%)^{*}$ & $2(3.33 \%)^{*}$ & $9(15 \%)$ \\
Total & $33(55 \%)$ & $27(45 \%)$ & $60(100 \%)$ \\
\hline
\end{tabular}

${ }^{*} \chi^{2}(1)=2.220, \mathrm{p}>0.05$ 
Table 3. Lithium and Dementia, Women only.

\begin{tabular}{cccc}
\hline & Lithium non user & Lithium user & Total \\
\hline No Dementia & $50(57.47 \%)^{*}$ & $21(24.1 \%)^{*}$ & $71(81.57 \%)$ \\
Dementia & $9(10.34 \%)^{*}$ & $7(8 \%)^{*}$ & $16(18.33 \%)$ \\
Total & $59(67.81 \%)$ & $28(32.1 \%)$ & $87(100 \%)$ \\
\hline
\end{tabular}

${ }^{*} \chi^{2}(1)=1.202, \mathrm{p}>0.05$

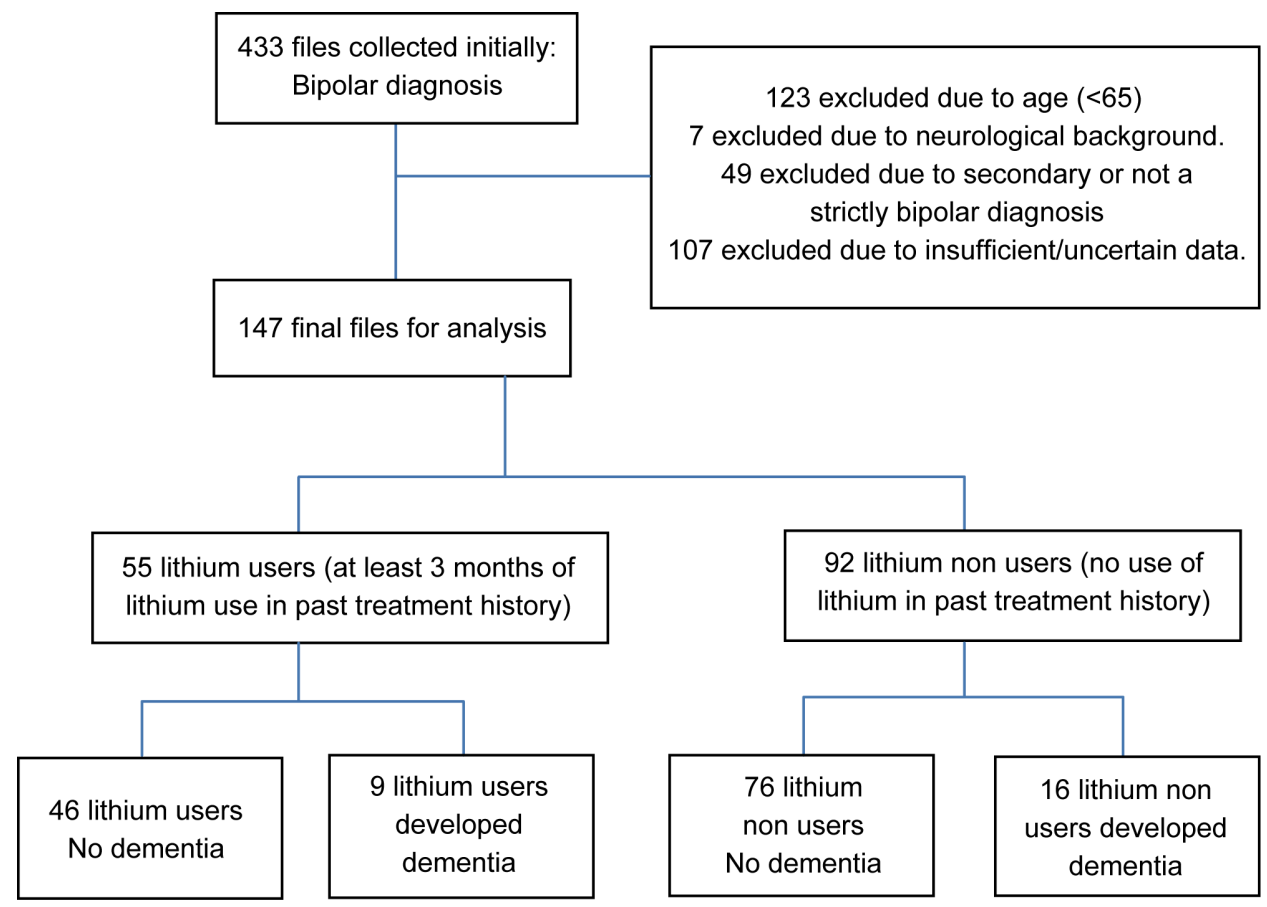

Figure 1. Flow chart-research population.

\section{Discussion}

The goal of this research was to discern whether lithium has protective effects on the development of dementia in a narrowly defined group of patients, namely-elderly patients with a strictly bipolar diagnosis treated with lithium for a period of at least 3 months. Our results show that lithium usage at any point in the patient's treatment history has no effect on dementia incidence in the elderly bipolar population. There was no statistically significant difference between lithium users and lithium non users.

In reviewing prior research, we found conflicting results regarding the neuroprotective qualities of lithium treatment-most of the research done in the field concluded that lithium provides neuroprotection against AD [9] [10] [11] [12] [14]. Yet two works showed opposed results:

1) A case control research by Dunn et al. (2005) investigated the correlation between lithium and dementia using the GP's Research Database in the UK. Their conclusions were that not only isn't lithium a neuroprotector, but that it might in fact increase dementia. However, the number of lithium users was small (87 patients), and there was a 
significant age difference between the cases and controls (9 years) [8].

2) The Hampel group performed a single blinded randomized control test (2009). For a period of 10 weeks, a group of 71 patients suffering of $\mathrm{AD}$ were given either lithium or placebo, and various markers were used to check for progress of the disease (Phosphorylated tau protein concentration in the CSF, GSK-3 activity and the MMSE, ADAS-Cog and NPI tests were used for neuropsychological and neuropsychiatric assessment. The research showed no significant difference on any of the tested values between the two groups, and concluded that lithium seems to have no effect [13].

Based upon the accumulated data we propose an alternative explanation. We suggest that usage of lithium confers limited time neuroprotection, which appears to last for a relatively short period of time (possibly months) and discontinuation of treatment causes loss of this effect.

The most similar research to our own was the Nunen et al. trial from 2007 [10]. That research, however, came to different conclusions than our own, showing that lithium does have neuroprotective qualities. Yet there are some differences in the research design, which can explain the different results: The Nunen research compared two groups, which differ from our own-patients who used lithium recently (during the last 6 months) versus those who haven't been using it in the recent past (Yet may have used it prior to the last 6 months). The latter group was subdivided into people who have received lithium in the past, (before 6 months) and patients who never used lithium in their lives. Concerning these last two groups (People who have either used lithium prior to 6 months, or who have never used lithium) they mention that the rates of dementia are quite similar in the two groups, with no significant difference as well. These two groups are similar to our researched groups (Since in our definitions lithium users were patients who used lithium for at least 3 months, at any time in their treatment history, but without consideration for how recent the treatment was). Our research provides more statistical strength to this analysis, due to the larger sample size of our research (The Nunen research had only 33 patients in the two groups mentioned, while we had 147). Together with the Nunen research, our research strengthens the evidence that prior, non-recent lithium use has no real effect on dementia incidence rates.

The results of the Forlenza randomized control test from 2012 [14] show supporting evidence-that lithium reduces the markers for mild cognitive impairment (MCI) and lowers the incidence of $\mathrm{AD}$, after short term continuous use (12 months in the research). We suggest a further research avenue-It may be of interest to explore the duration of effect of lithium, and the waning of its neuroprotective potential after cessation of use, and the length of recent lithium use needed to benefit from its neuroprotective capabilities.

Our research tried to explore the possible gender effect that lithium might have on dementia. Our results seem to indicate that in men lithium may have a slightly protective effect, while in women the opposite; however, both effects were not statistically significant. It may be worth to explore this finding in studies with greater numbers of subjects, for greater statistical power. 
Our research is not without limitations. Though one of its strong points is the welldefined and homogenous sample population (the elderly bipolar population), its results are not guaranteed to be relevant to a wider population. A possible problem with the analysis stems from the different sources of files-some came from the inpatient departments and others from the outpatient clinics thus some lacked detailed history of past medication use or specific duration of lithium use ( 107 had to be excluded due to insufficient/uncertain data).

\section{Acknowledgements}

We thank Ms. Hana Zemishlany and Mr. Alexei Sigalov for helping with data collection and blinding the files.

This work was done by Dr. Meir Dvir in partial fulfillment of the M.D. thesis requirements of the Sackler Faculty of Medicine, Tel Aviv University.

\section{Financial and Material Support}

Meir Dvir, Shelly Tadger and Asaf Shelef have nothing to declare.

Diana Paleacu Kertesz has received consulting fees, lecture fees from Novartis and participated in paid advisory boards for Novartis and Samuelov Pharmaceuticals.

This study has not been funded.

\section{References}

[1] Smith, L.A., Victoria, C. and Warnock, A. (2007) Effectiveness of Mood Stabilizersand Antipsychotics in the Maintenance Phase of Bipolar Disorder: A Systematic Review of Randomized Controlled Trials. Bipolar Disorders, 9, 394-412. http://dx.doi.org/10.1111/j.1399-5618.2007.00490.x

[2] Geddes, J.R., Burgess, S. and Hawton, S. (2004) Long-Term Lithium Therapy for Bipolar Disorder: Systematic Review and Meta-Analysis of Randomized Controlled Trials. American Journal of Psychiatry, 161, 217-222. http://dx.doi.org/10.1176/appi.ajp.161.2.217

[3] Spires, T.L. and Hyman, B.T. (2005) Transgenic Models of Alzheimer's Disease: Learning from Animals. NeuroRx, 2, 423-437. http://dx.doi.org/10.1602/neurorx.2.3.423

[4] Camins, A., Verdaguer, E. and Junyent, F. (2009) Potential Mechanisms Involved in the Prevention of Neurodegenerative Diseases by Lithium. CNS Neuroscience \& Therapeutics, 15, 333-348. http://dx.doi.org/10.1111/j.1755-5949.2009.00086.x

[5] Sofola, O., Kerr, F. and Rogers, I. (2000) Inhibition of GSK-3 Ameliorates Ab Pathology in an Adult-Onset Drosophila Model of Alzheimer's Disease. PLoS Genetics, 6, e1001087. http://dx.doi.org/10.1371/journal.pgen.1001087

[6] Lautebach, E.C., Victoroff, J. and Coburn, K.L. (2010) Psychopharmacological Neuroprotection in Neurodegenerative Disease: Assessing the Preclinical Data. Journal of Neuropsychiatry \& Clinical Neurosciences, 8-18. http://dx.doi.org/10.1176/jnp.2010.22.1.8

[7] Forlenza, O.V., Paula, V.J. and Machado-Vieira, R. (2012) Does Lithium Prevent Alzheimer's Disease? Drugs Aging, 29, 335-344.

http://dx.doi.org/10.2165/11599180-000000000-00000

[8] Dunn, N., Holmes, C. and Mullee, M. (2005) Does Lithium Therapy Protect against the Onset of Dementia? Alzheimer Disease \& Associated Disorders, 19, 20-22. 
http://dx.doi.org/10.1097/01.wad.0000155068.23937.9b

[9] Terao, T., Nakano, H. and Inoue, Y. (2006) Lithium and Dementia: Apreliminary Study. Progress in Neuro-Psychopharmacology \& Biological Psychiatry, 30, 1125-1128. http://dx.doi.org/10.1016/j.pnpbp.2006.04.020

[10] Nunes, P.V., Forlenza, O.V. and Gattaz, W.F. (2007) Lithium and Risk for Alzheimer's Disease in Elderlypatients with Bipolar Disorder. British Journal of Psychiatry, 190, 359360. http://dx.doi.org/10.1192/bjp.bp.106.029868

[11] Kessing, L.V., Sønderga, L. and Forman, J.L. (2008) Lithium Treatment and Risk of Dementia. Archives of General Psychiatry Journal, 65, 1331-1335. http://dx.doi.org/10.1001/archpsyc.65.11.1331

[12] Kessing, L.V., Sønderga, L., Forman, J.L. and Andersen, P.K. (2010) Does Lithium Protect against Dementia? Bipolar Disorders, 12, 87-92. http://dx.doi.org/10.1111/j.1399-5618.2009.00788.x

[13] Hampel, H., Ewers, M. and Bürger, K. (2009) Lithium Trial in Alzheimer's Disease: A Randomized, Single-Blind, Placebo-Controlled, Multicenter 10-Week Study. Journal of Clinical Psychiatry, 70, 922-931. http://dx.doi.org/10.4088/JCP.08m04606

[14] Forlenza, O.V., Diniz, B.S., Radanovic, M. and Santos, F.S. (2011) Disease Modifying Properties of Long-Term Lithium Treatment for Amnestic Mild Cognitive Impairment: Randomized Controlled Trial. The British Journal of Psychiatry, 198, 351-356.

http://dx.doi.org/10.1192/bjp.bp.110.080044

Submit or recommend next manuscript to SCIRP and we will provide best service for you:

Accepting pre-submission inquiries through Email, Facebook, LinkedIn, Twitter, etc.

A wide selection of journals (inclusive of 9 subjects, more than 200 journals)

Providing 24-hour high-quality service

User-friendly online submission system

Fair and swift peer-review system

Efficient typesetting and proofreading procedure

Display of the result of downloads and visits, as well as the number of cited articles

Maximum dissemination of your research work

Submit your manuscript at: http://papersubmission.scirp.org/

Or contact aad@scirp.org 